\title{
Fiber-optic Raman Probe Coupled with a Ball Lens for Improving Depth-resolved Raman Measurements of Epithelial Tissue: Monte Carlo Simulations
}

\author{
Zhiwei Huang \\ Optical Bioimaging Laboratory, Department of Bioengineering, \\ Faculty of Engineering, National University of Singapore, \\ Singapore
}

\section{Introduction}

Raman spectroscopy is a vibrational spectroscopic technique capable of optically probing biomolecular changes of tissue associated with neoplastic transformation, and has shown promise for the noninvasive, in vivo diagnosis and detection of epithelial precancer and cancer in various organs [1-3]. But analyzing Raman spectroscopic information emitted from tissue remains complicated for tissue diagnosis and characterization due to the reasons that the overall Raman signals acquired from the tissue surface usually contain a mixture of Raman information originating from different tissue depths [4, 5], while the changes of tissue morphology or biochemical constituents associated with disease transformation may be depth-dependent in biomedical systems [6]. For instance, the epithelial tissue usually consists of a superficial epithelium and an underlying stroma, the dysplasia-related changes (precancer) may be associated with the thickening of epithelial tissue, which results in the attenuation of the excitation light to penetrate into deeper areas of tissue and also the attenuation of Raman emission from deeper tissue regions (e.g., stroma) [7, 8]. On the other hand, the changes of other optical properties (e.g., absorption coefficient, scattering coefficient, anisotropic factor, refractive index) of tissue are also correlated with tissue physiologic/pathologic status, significantly affecting the overall Raman signal collection from biomedical tissue [9]. Hence, to better understand the origins of Raman signals collected from tissue surface for further improving the diagnosis of epithelial precancer or early cancer, it is highly desirable to develop a depth-resolved Raman spectroscopic technique for facilitating the wide applications of Raman spectroscopy in biomedical diagnosis.

A number of fiber-optic probe designs have been reported for depth-resolved optical spectroscopic measurements, but most work are centered on fluorescence and reflectance spectroscopy for tissue diagnosis [10-15]. The depth-resolved fiber probe designs can mainly be classified into two types: (i) single-fiber probe in which the same fiber is used for both light excitation and reflectance/fluorescence/Raman collection, and (ii) multiple-fiber probe 
in which separate fibers are used for light delivery and reflectance/fluorescence/Raman collection [8-12]. Although the single-fiber probe with a smaller aperture (i.e., core diameter) shows a high sensitivity of detecting spectral signal particularly emitted from superficial layer of epithelial tissue, its collection efficiency is much reduced when the fiber diameter decreases. With multiple-fiber probe designs, varying the excitation-collection fiber separations or the oblique angles between the excitation-collection fibers permit the depthdiscrimination of fluorescence/Raman signals from tissue [11, 12]. Although the multi-fibers probe design has shown a better depth-selectivity for spectroscopic acquisitions, the bulky probe design is unsuited for endoscope-based biomedical applications. In this work, we propose a fiber-optic Raman probe design coupled with a ball lens for improving the collection efficiency of depth-resolved Raman measurements, particularly for probing subsurface regions of epithelial tissue. The use of ball lens as the illumination and collection optics of spectroscopic probes has proven to be efficient for epithelial tissue measurement [16]. Moreover, ball-lens probe shows advantages of compactness and short working distance which are in favor of in vivo tissue measurement. In this study, we employ the Monte Carlo (MC) simulation method to evaluate the performance of the ball-lens Raman probe designs under different optical configurations. Raman experiments of a ball lens Raman probe design on a layered tissue phantom are also tested.

\section{Materials and methods}

\subsection{Raman probe configuration}

Fig. 1 shows the schematic of a fiber-optic Raman probe design coupled with a ball lens for depth-resolved tissue Raman measurements [8]. The laser light can be coupled into the excitation arm of the Raman probe through a single fiber $(200 \mu \mathrm{m}, \mathrm{NA}=0.22)$, and delivered

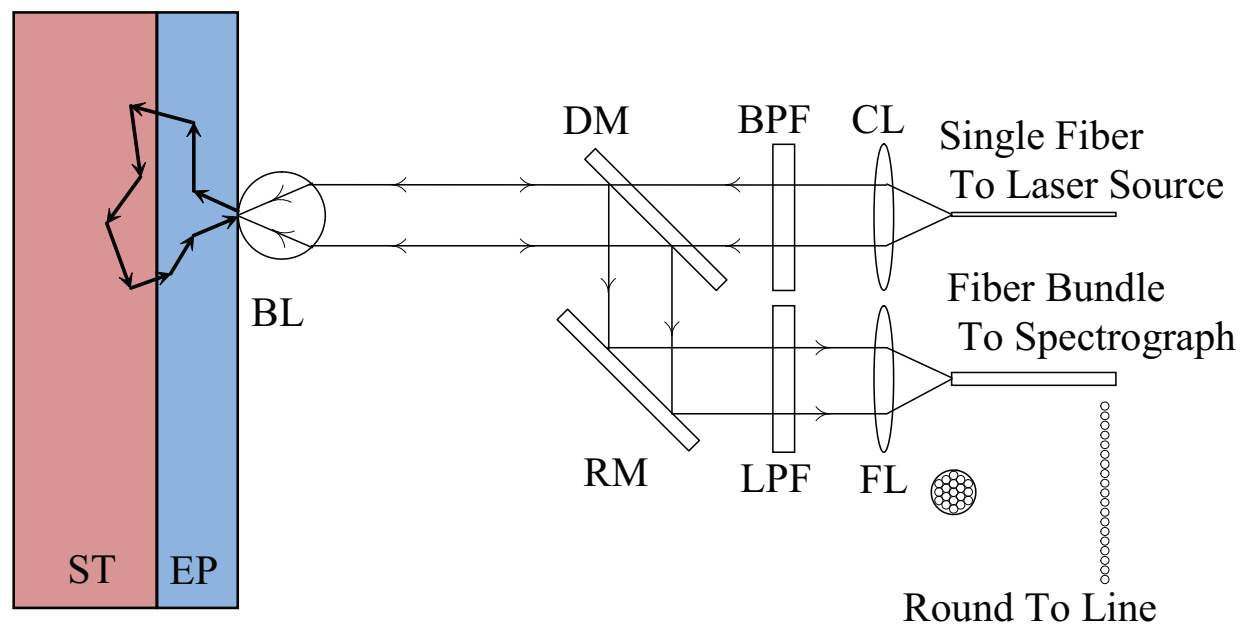

Fig. 1. Schematic of the Raman probe design and two-layer epithelial tissue model for Monte Carlo (MC) simulations. CL: collimating Lens; FL: Focusing Lens; BPF: Band Pass Filter; LPF: Long Pass Filter; DM: Dichroic Mirror; RM: Reflection Mirror; BL: Ball Lens; EP: Epithelium; ST: Stroma. 
into the filtering module incorporated with a near-infrared (NIR) lens for collimating excitation light and a narrow band-pass (BP) filter for removing fiber fluorescence and laser noise. Then, the laser light is focused onto the tissue through a ball lens mounted on the tip of the Raman probe. The backscattered light is collected by the same ball lens and subsequently reflected back into the collection arm of the Raman probe through a dichroic mirror and a reflection mirror. The scattered light can be further filtered by an edge longpass (LP) filter to block the Rayleigh scattered laser light while allowing Raman scattered light photons to get into the collection fiber bundles $(28 \times 50 \mu \mathrm{m}$ arranged in line, NA=0.22) for tissue Raman signals detection [3].

\subsection{Monte Carlo simulations}

Monte Carlo simulations have been widely used to mimic light propagation in turbid media (e.g., biological tissue) [14, 15, 17], and also simulate the fluorescence generation and propagation in biological tissue [14, 15, 17-19]. With the development of MC simulation model to evaluate the fiber probe design coupled with a ball lens for depth-resolved fluorescence measurement on layered epithelial tissue [14, 15], we further modified the MC model to study Raman light generation and propagation in epithelial tissue as well as Raman signal collection with the use of ball lens fiber-optic Raman probe. The modified MC codes include the following modules: (i) excitation light propagation in ball lens and tissue; (ii) Raman photons generation and propagation in tissue; (iii) Raman photons collection by the ball lens and propagation in the ball lens; (iv) Raman photons collection by the focusing lens and subsequently by the collection fibers of the Raman probe.

In MC simulations, one hundred million excitation light photons are launched into the tissue through the ball lens. The photons are initialized as a uniform light beam with a beam diameter the same as the ball lens' diameter. When the excitation light photons are incident into the ball lens or the Raman photons pass through the ball lens, the direction of the refracted light is calculated using Snell's law. The photon weight is then updated as $\mathrm{w} \leftarrow \mathrm{w}(1-R)$, whereby the reflection coefficient, $R$, is calculated using Fresnel's formula:

$$
R=\frac{1}{2}\left[\frac{\sin ^{2}\left(\alpha_{i}-\alpha_{t}\right)}{\sin ^{2}\left(\alpha_{i}+\alpha_{t}\right)}+\frac{\tan ^{2}\left(\alpha_{i}-\alpha_{t}\right)}{\tan ^{2}\left(\alpha_{i}+\alpha_{t}\right)}\right]
$$

where $\alpha_{i}$ and $\alpha_{t}$ are the incident angle and the refracted angle, respectively. In this simulation, Raman generation is estimated with the probability $(p)$ of the illumination light to be converted to Raman scattered light as follows [20,21]:

$$
p=\left(\frac{\mu_{s}}{\mu_{a}+\mu_{s}}\right) \times Q Y
$$

where $\mu_{a}$ is the absorption coefficient for generating Raman photons, $\mu_{s}$ is the scattering coefficient, and QY $(<<1)$ is the Raman quantum yield. To determine the Raman photon generation at a particular scattering position, a random number between 0 and 1 is generated; if the probability yielded in Eq. (2) is larger than the random number, then Raman photon is produced; otherwise, no Raman photon is generated. In our MC programs, we presume that the radiation angle of Raman photons emitted from the Raman scatterers is isotropic [20] and the emitted Raman photons will not be reabsorbed by the Raman scatterers for generating secondary emission of Raman photons due to their extremely low 
intensity levels. The deflection angle $(\beta)$ of each scattering in the medium is described by Henyey-Greenstein phase function as the following equation [17, 22]:

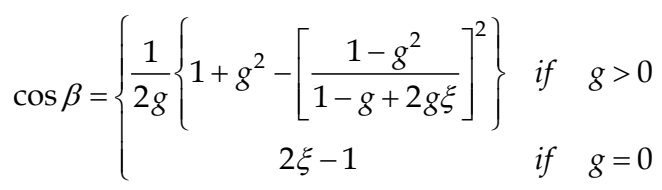

where $\mathrm{g}$ is anisotropy factor and $\xi$ is a random number between 0 and 1 . The azimuthal angle $\psi$ can be determined by $2 \Pi \xi$.

After escaping from tissue surface, Raman photons are collected by the ball lens within the collection cone angle [23]:

$$
\theta=\sin ^{-1}\left(\frac{2\left(n_{b}-1\right)}{n_{b} n_{t}}\right)
$$

where $\theta$ is half-angle of the collection cone of the ball lens; $n_{b}$ and $n_{t}$ are the refractive indices of the ball lens and tissue, respectively. Tissue Raman photons which pass through the ball lens will be coupled into the collection fibers by a focusing lens (focal length, $f=12 \mathrm{~mm}$ ) of the Raman probe. Eventually, all those photons that reach the collection fibers within the collection cone angle of the collection fibers $(\mathrm{NA}=0.22)$ will be considered as the collected Raman photons by the Raman probe. The 2-D distributions of excitation light and the origins of the Raman photons collected from tissue will be given by MC simulations to evaluate the performance of the fiber-optic Raman probe design for depth-resolved tissue Raman measurements. In addition, the collection efficiency of the Raman probe defined as the ratio of the collected tissue Raman photon numbers to the incident excitation light photon numbers is also estimated by using MC simulations.

\subsection{Two-layer epithelial tissue model}

A two-layer tissue model which mimics the epithelial tissues is used for MC simulations of the excitation light and Raman photons propagation in tissue. Table 1 gives the optical properties (absorption coefficient, $\mu_{a}$; scattering coefficient, $\mu_{s}$; anisotropy factor, g; tissue refractive index, $n_{t}$, and tissue thickness, d) [24] of the modeled tissue. The wavelengths of excitation light and Raman scattered photon light are selected at 785 and $820 \mathrm{~nm}$, respectively, for MC simulations [2, 3].

\begin{tabular}{c|c|c|c|c|c|c}
\hline Tissue Layer & Wavelength $(\mathrm{nm})$ & $\mu_{a}\left(\mathrm{~cm}^{-1}\right)$ & $\mu_{s}\left(\mathrm{~cm}^{-1}\right)$ & $g$ & $n_{t}$ & $\mathrm{~d}(\mathrm{~mm})$ \\
\cline { 1 - 5 } Epithelium & $\begin{array}{c}785 \\
\text { Stroma }\end{array}$ & 0.9 & 25 & 0.9 & 1.4 & 0.3 \\
\cline { 1 - 5 } Epithelium & $\begin{array}{c}820 \\
\text { (Raman) }\end{array}$ & 0.45 & 165 & 0.9 & 1.4 & 3 \\
\cline { 1 - 6 } Stroma & (Ration) & 0.3 & 150 & 0.9 & 1.4 & 3 \\
\hline
\end{tabular}

Table 1. Optical properties of the two-layer epithelial tissue model for MC simulations $[14,15,24]$. 


\section{Simulation results and discussion}

\subsection{Effect of the refractive index of the ball lens on tissue Raman measurements}

Fig. 2 shows the MC simulation results of the $785 \mathrm{~nm}$ excitation light distribution in epithelial tissue for the fiber-optic Raman probe designs with different refractive indices of the ball lens and a fixed diameter of $3 \mathrm{~mm}$. Note that the indices selected for simulation correspond to different optical materials (1.46-UV fused silica; 1.51-Boro-crown glass; $1.63-$ Dense flint glass; 1.76-Sapphire; 1.83-Lanthanum flint glass). Overall, the excitation light exhibits higher intensity over the whole tissue when increasing the refractive index of the ball lens. A 1.1- to 1.5- fold increase in excitation intensity distribution in tissue is found for the use of ball lens at refractive indices of 1.51, 1.63, 1.76 and 1.83 as compared to the refractive index of 1.46. The corresponding excitation light distribution in the epithelium is approximately 1.1- to 2.7- fold stronger. This suggests that Raman probe with a larger refractive index of the ball lens achieves higher efficiency in focusing the excitation light photons into the tissue, especially in the epithelium layer of the tissue.
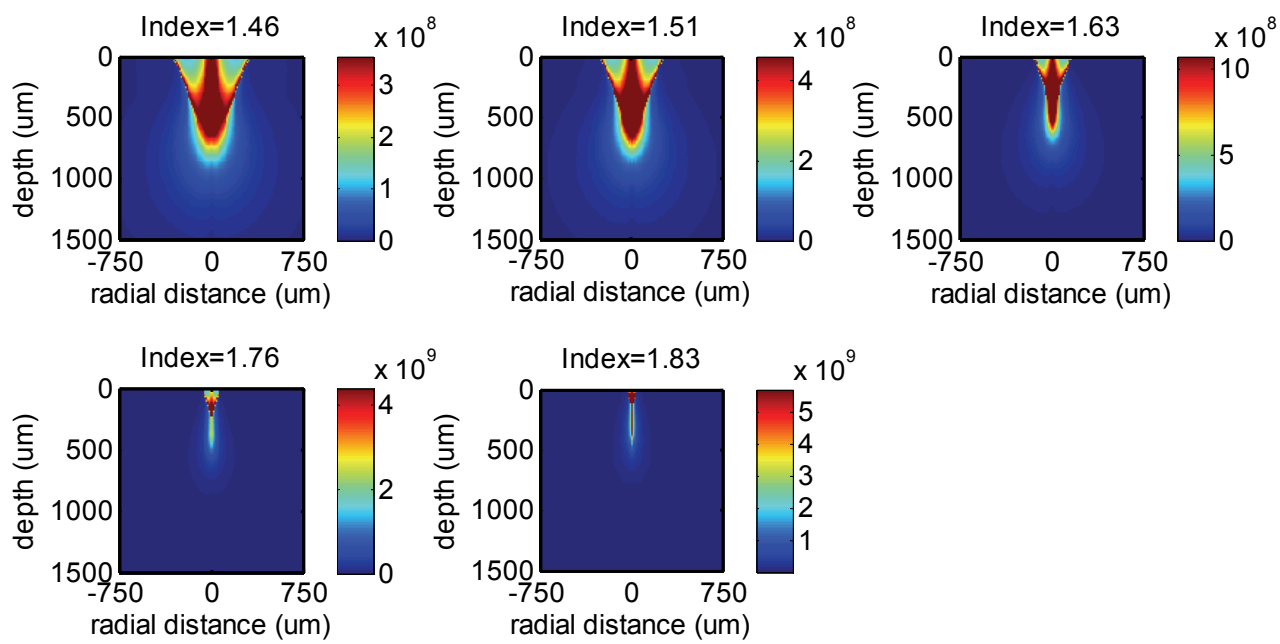

Fig. 2. The 785-nm excitation light distributions along the tissue depth and radial directions in tissue using the Raman probe designs with different refractive indices of the ball lenses $(n=1.46,1.51,1.63,1.76$ and 1.83).

Fig. 3 shows the 785-nm excitation light distributions along the tissue depth which are the sum of the excitation light distribution (Fig. 2) over the radial dimension at each tissue depth. Each depth profile is normalized to the integrated area under the profile for better comparison among different refractive indices of the ball lens in Raman probe. The excitation light undergoes a shorter penetration into tissue when increasing the refractive index of the ball lens. For instance, an intensity maximum of the excitation light in epithelium layer is observed around the focal plane of the ball lens when using the refractive index of 1.76 or 1.83 of the ball lens (Fig. 3). This suggests that increasing the refractive index of the ball lens of the Raman probe design makes the excitation light to be more tightly focused in the shallower regions of tissue (i.e., the epithelium). The findings 
above can be explained by the enhanced focusing ability of the ball lens attributed to the increase of refractive index. The ball lens focal length can be estimated using the following equations [23]:

$$
B F L=E F L-\frac{D}{2} ; \quad E F L=\frac{n_{b} D}{4\left(n_{b}-1\right)}
$$

where BFL is back focal length, representing the distance from the ball lens front tip to the focal point; EFL is effective focal length, representing the distance from the ball lens center to the focal point; $\mathrm{D}$ and $n_{b}$ is the diameter and the refractive index of the ball lens, respectively. According to Eq. (5), if keeping diameter D constant, EFL and BFL are inversely proportional to refractive index $n_{b}$. That is why the ball lens with a larger refractive index leads to a shorter light penetration depth in tissue

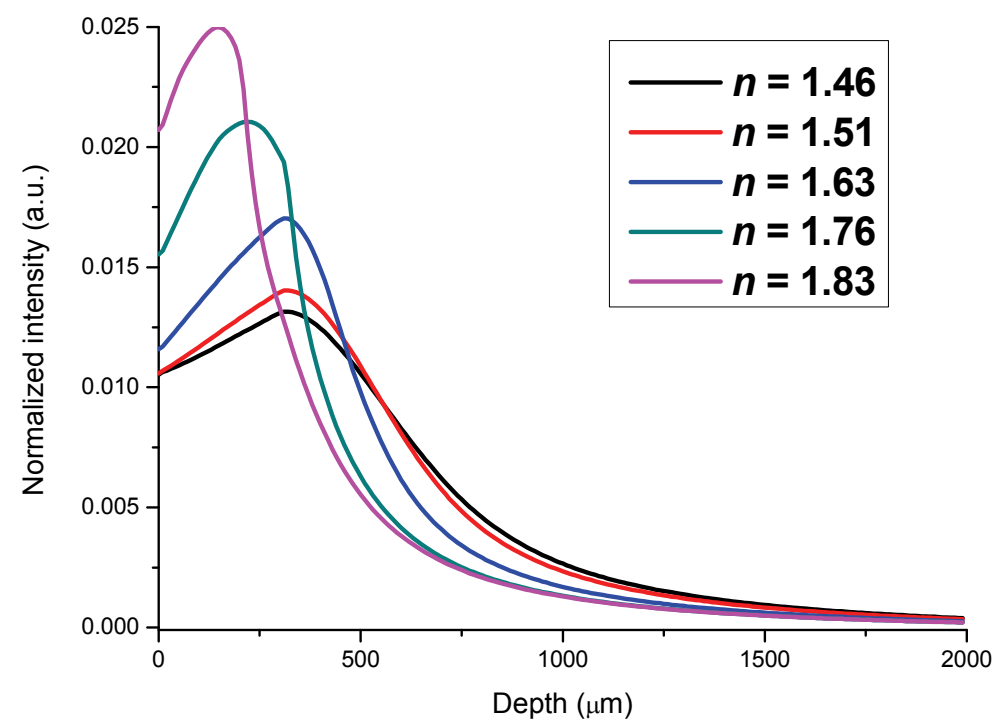

Fig. 3. The 785-nm excitation light distributions along the tissue depth using the Raman probe designs with different refractive indices of the ball lenses ( $n=1.46,1.51,1.63,1.76$ and 1.83).

Fig. 4 shows the calculated results of the origins of the Raman photons generated from different tissue locations of epithelial tissue. Similar to the excitation light distribution in tissue (Fig. 2), the Raman photons generated from different tissue depths are also dependent on the use of the refractive index of the ball lens in Raman probe designs. With the increase of the refractive index of the ball lens, Raman photons radiation tends to be more strongly coming from the shallower regions of the epithelium layer (e.g., $\sim 5$ orders of magnitude stronger in Raman intensity using the ball lens's refractive index of 1.83 as compared to that using the ball lens's refractive index of 1.46) (Fig. 4). This is due to the fact that excitation light is more tightly focused in the shallower layer of the epithelium, and the ball lens with a larger refractive index has larger collection cone angle (Eq. (4)), leading to the significant improvements of Raman signal collections from the epithelium in tissue. The findings above 
imply that increasing the refractive index of the ball lens in Raman probe design can effectively improve the collection efficiency of tissue Raman photons from the tissue, especially from the subsurface regions of the epithelium layer in tissue.
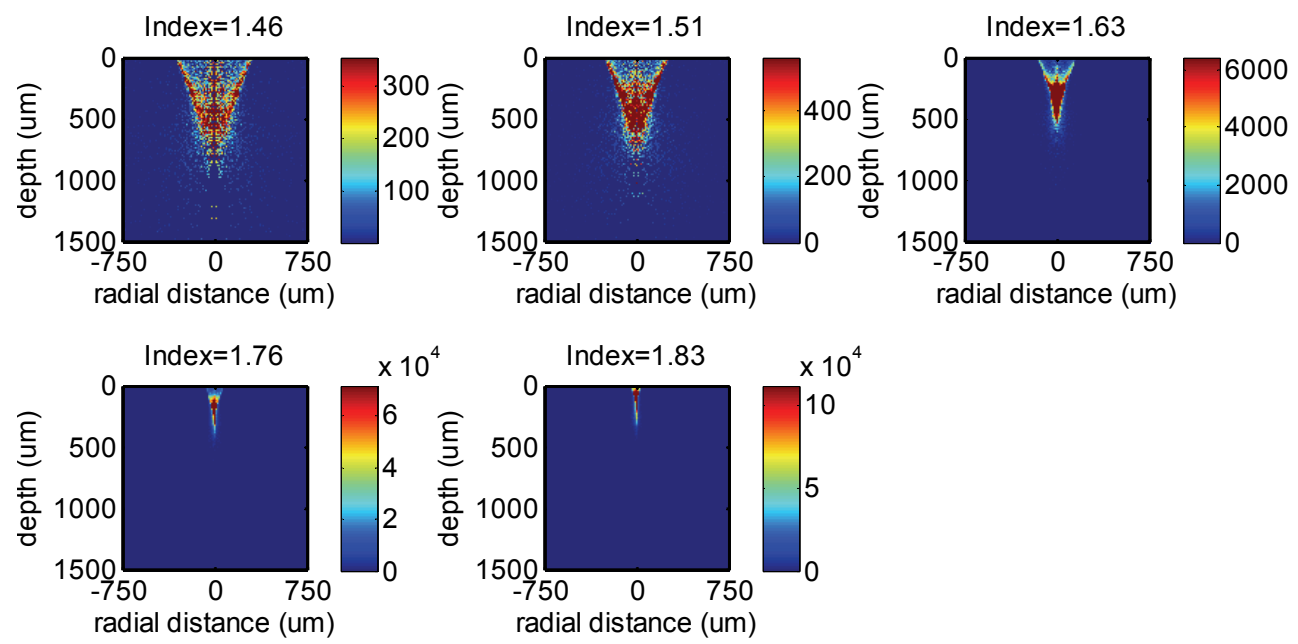

Fig. 4. Distributions of the Raman photons collected from different tissue depths using the Raman probe designs with different refractive indices of the ball lens $(n=1.46,1.51,1.63,1.76$ and 1.83).

Fig. 5 shows the depth distribution of the origins of Raman photons collected from tissue for the use of different refractive indices of the ball lens in Raman probe designs. The depth distribution is created by integrating the 2-D distribution (Fig. 4) over the radial dimension at each tissue depth and is then normalized to the integrated area under the profile. It can be used to represent the relative contribution of Raman photons from different tissue depths to the overall collected Raman photons. The relatively weak Raman photons are generated throughout different tissue depths when using the ball lens with refractive indices of less than 1.63 (Fig.5); but stronger Raman photons generation tends to be arising from the epithelium layer in tissue when using the ball lens with larger refractive indices (e.g., $\mathrm{n}=1.76,1.83$ ). For instance, a maximum Raman radiation appears in the shallower region $(\sim 40 \mu \mathrm{m})$ of the epithelium layer in tissue for the use of ball lens with refractive index of 1.83 (Fig. 5). This indicates that the Raman probe design with the use of high refractive index ball lens is in favor of detecting Raman signal emitted from subsurface layer of the epithelial tissue.

We also evaluate the collection efficiency of the Raman probe versus the refractive index of the ball lens (Fig. 6). The collection efficiency of the Raman probe increases monotonously when increasing the refractive index of 1.46 up to 1.83. All the MC simulation results above confirm that Raman probe design with the use of a larger refractive index of the ball lens has the advantage of preferentially detecting the Raman signal from subsurface regions of the epithelium in tissue with high collection efficiency. 


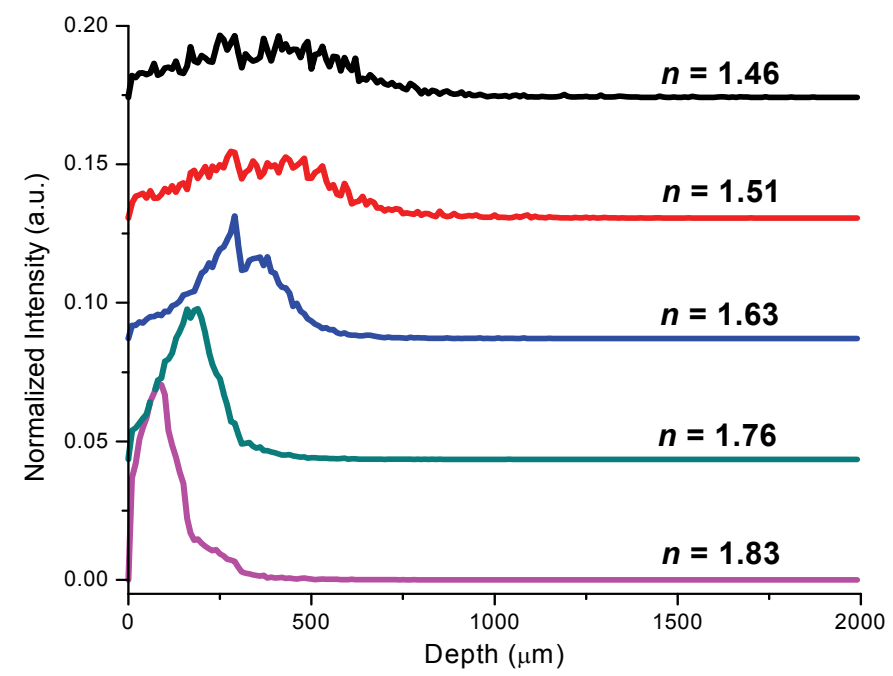

Fig. 5. Depth-resolved Raman photons collected from different tissue depths using the Raman probe designs with different refractive indices of the ball lens $(n=1.46,1.51,1.63,1.76$ and 1.83). Note that for comparison purpose, the depth-resolved Raman intensity profiles with different refractive indices of the ball lens have been vertically shifted to different intensity levels.

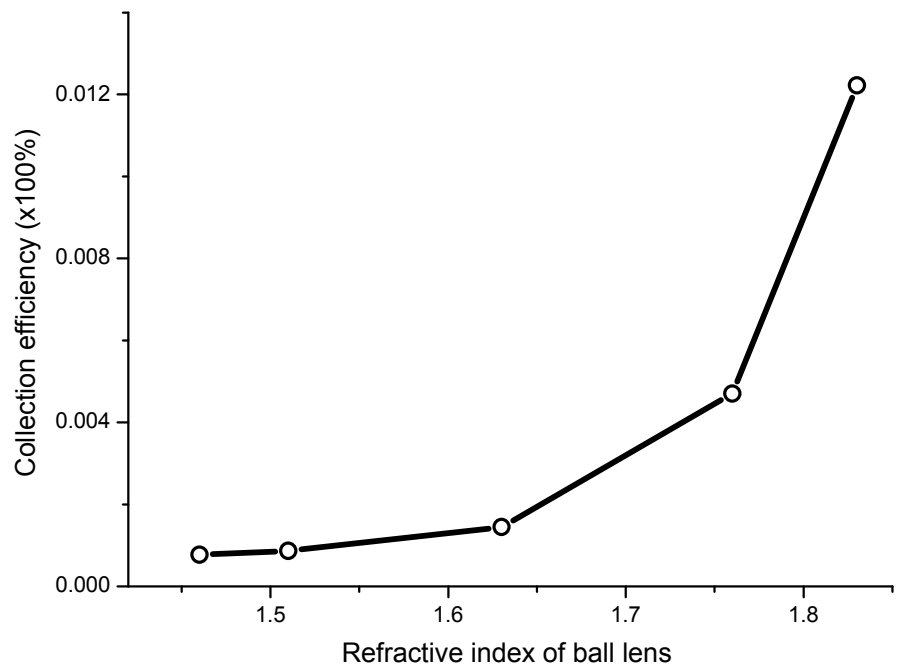

Fig. 6. Collection efficiency of the Raman probe as a function of the refractive index of the ball lens.

\subsection{Effect of the ball lens diameter on tissue Raman measurements}

Fig. 7 shows the MC simulation results of the $785 \mathrm{~nm}$ excitation light distribution in epithelial tissue for the fiber-optic Raman probe designs with different diameters of the ball lens (refractive index of 1.83). With the use of smaller diameters of the ball lens in Raman 

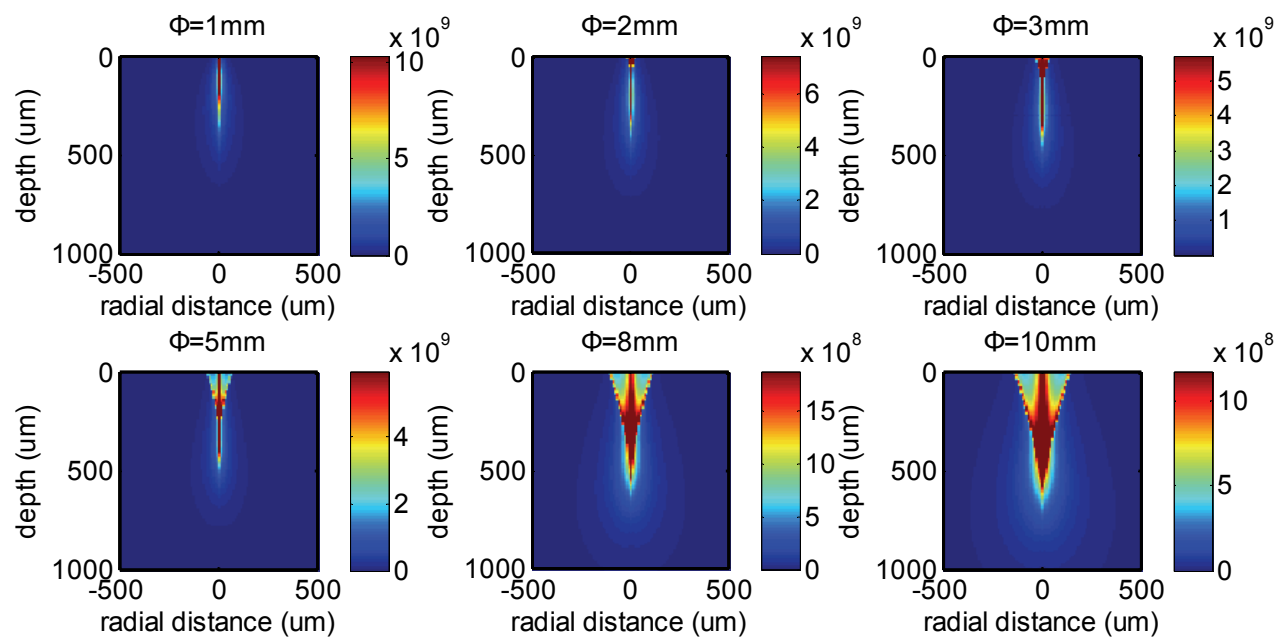

Fig. 7. The 785-nm excitation light distributions in tissue using the Raman probe designs with different diameters of the ball lens $(\Phi=1,2,3,5,8$ and $10 \mathrm{~mm})$.

probe design, the incident light is found to be more tightly focused into shallower regions of epithelial tissue with stronger incident light distribution as compared to the use of large diameters of the ball lens. This can be explained by the shortening of the ball lens focal length (Eq.5) [23] when using the ball lens with small diameters. Fig. 8 presents the depth distribution of the $785 \mathrm{~nm}$ excitation light in tissue calculated by integrating the 2-D intensity distribution (Fig. 7) over the radial direction at each tissue depth. The excitation light from the Raman probe appears to have a shorter penetration depth in tissue when

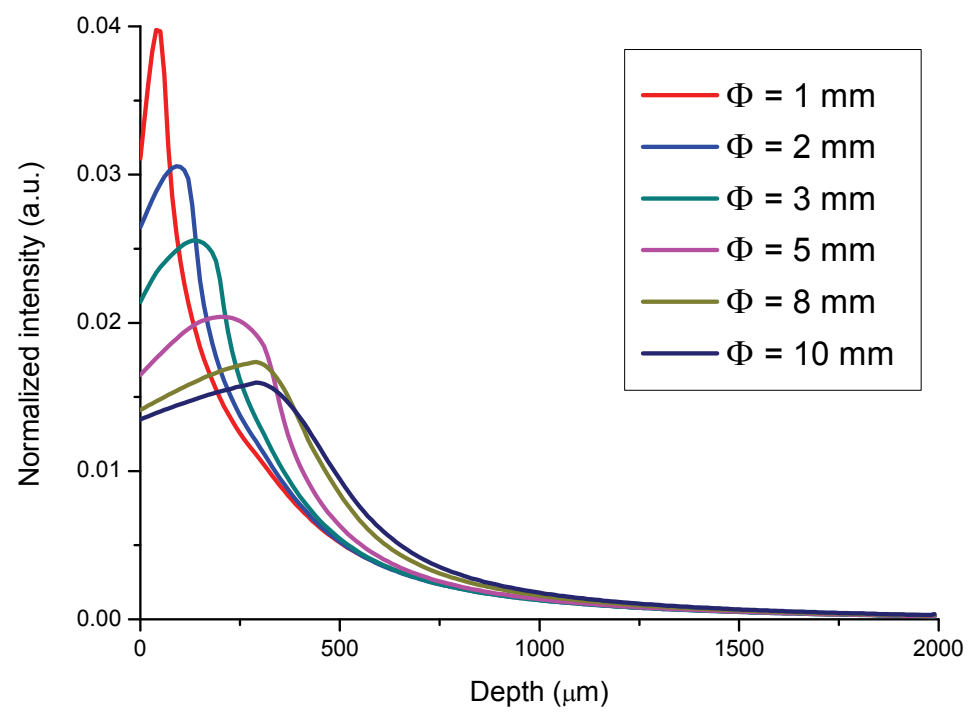

Fig. 8. The 785-nm excitation light distribution along the tissue depth using the Raman probe designs with different diameters of the ball lens $(\Phi=1,2,3,5,8$ and $10 \mathrm{~mm})$. 
using a smaller diameter of the ball lens. For instance, an incident light intensity maximum occurs at the subsurface region ( $\sim 50 \mathrm{um})$ of the epithelial tissue when the Raman probe uses the ball lens with a diameter of $1 \mathrm{~mm}$, whereas the incident light tends to penetrate into deeper regions of the epithelial tissue when using the ball lens with larger diameters (e.g., 10 $\mathrm{mm}$ ) (Fig. 8). Again, this implies that the Raman probe design with the use of smaller diameters of the ball lens can be more efficiently focusing the excitation light into the shallower regions of the epithelium layer in tissue.

Fig. 9 depicts the 2-D distributions of the origins of the Raman photons collected from tissue using the Raman probes with different diameters of the ball lens. Similar to the corresponding excitation light distribution in tissue (Fig. 7), the tissue Raman photons collected with the use of a small diameter of the ball lens in Raman probe design appears to be mostly originating from the shallower areas of the epithelium layer in tissue as compared to the Raman probe design with larger diameters of the ball lens. This can be more clearly displayed in the depth distribution of the origins of the Raman photons collected from tissue using the Raman probe designs with different diameters of the ball lens (Fig. 10). The relative contribution of the Raman photons from the epithelium becomes more dominant when reducing the ball lens' diameter in Raman probe design. For example, the Raman probe design with the use of $1 \mathrm{~mm}$ ball lens enables a detection of $\sim 95 \%$ of Raman signal arising from the epithelium, while only about $25 \%$ of Raman signal originating from the epithelium can be detected if using the $10 \mathrm{~mm}$ ball lens. Hence, the Raman probe design with a smaller diameter of ball lens can efficiently improve the detection of the Raman signal originating from the epithelium layer in tissue.

MC simulations are also carried out to exhaustedly search for all the possible combinations of the diameters and the refractive indices sof the optimized Raman probe designs for improving depth-resolved Raman measurements. Fig. 11 describes the depth-resolved measurement performance of the probe design as a function of the combination of the ball lens' diameter and refractive index. The results show that the ball lens with a refractive
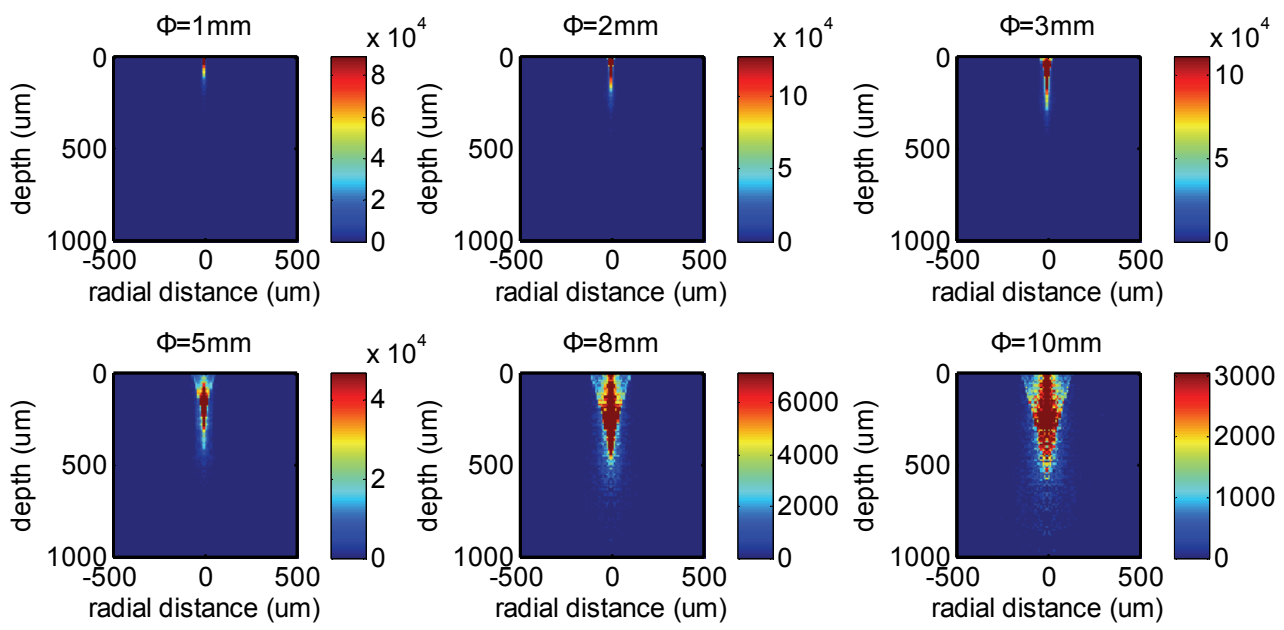

Fig. 9. Distributions of the origins of the Raman photons collected from tissue using the Raman probe designs with different diameters of ball lenses $(\Phi=1,2,3,5,8$ and $10 \mathrm{~mm})$. 


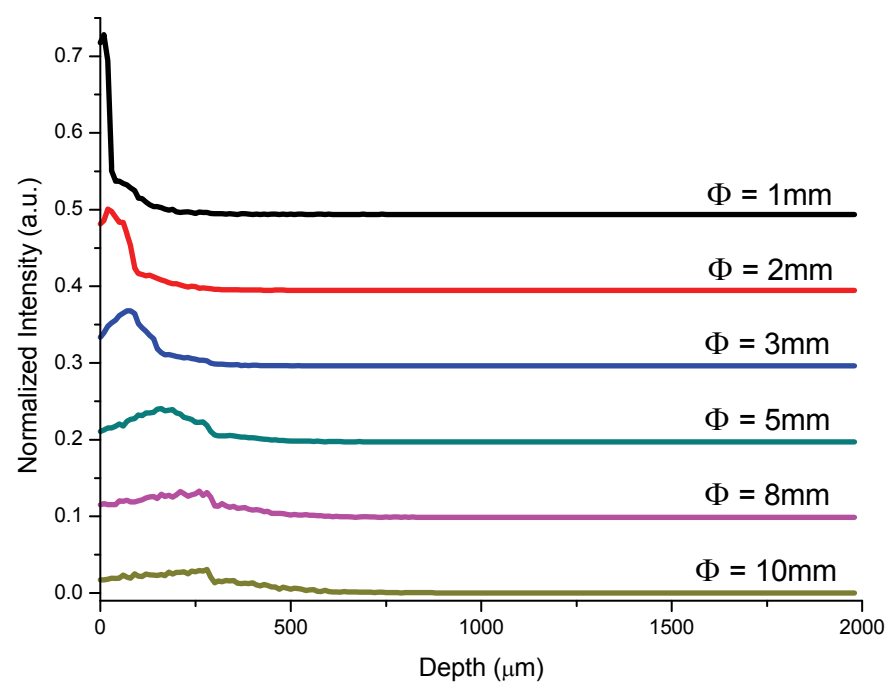

Fig. 10. Depth distribution of the origins of the Raman photons collected from tissue using the Raman probe designs with different diameters of the ball lens $(\Phi=1,2,3,5,8$ and $10 \mathrm{~mm})$.
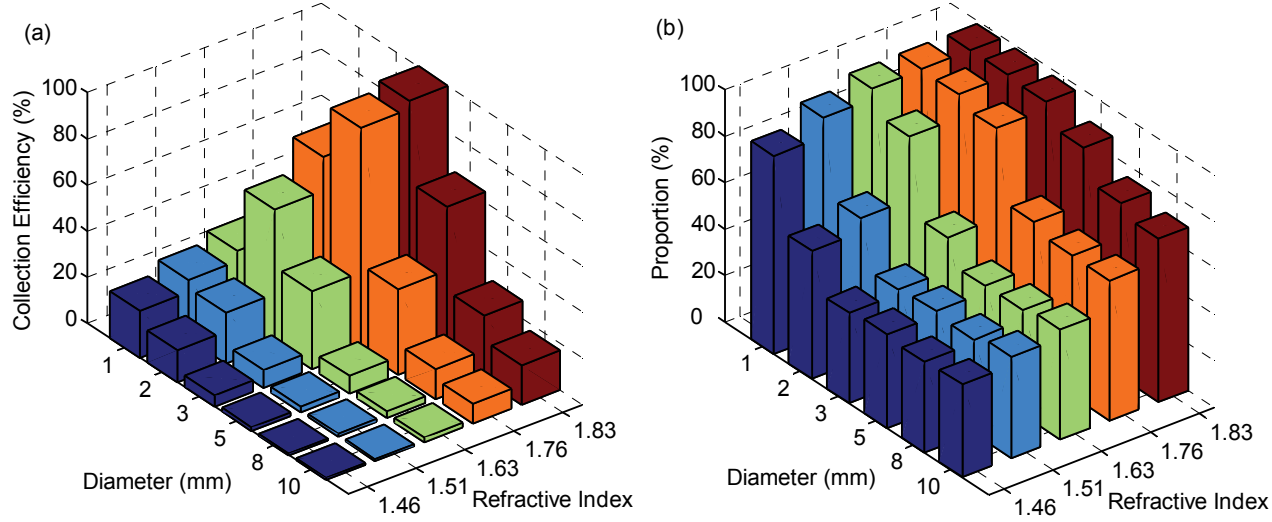

Fig. 11. The simulation results of the probe design based on various combinations of ball lens diameters (i.e., 1, 2, 3, 5, 8 and $10 \mathrm{~mm}$ ) and refractive indices (i.e., 1.46, 1.51, 1.63, 1.76 and 1.83): (a) collection efficiency; (b) proportion of the Raman photons from the epithelium to the total collected Raman photons from the tissue surface.

index of 1.83 and diameter of $3 \mathrm{~mm}$ achieves the highest collection efficiency (Fig. 11a). With the use of this probe design, Raman photons originating in the epithelium of tissue cover 97\% of the overall Raman photons collected from the surface of epithelial tissue as shown in Fig. 11b, indicating a good depth-selectivity to the epithelium. Although the depthselectivity can be further improved by reducing the diameter of the ball lens down to $2 \mathrm{~mm}$, the collection efficiency will drop by about $40 \%$. This suggests that the Raman probe design with the use of a ball lens with the diameter of $3 \mathrm{~mm}$ and the refractive index of 1.83 is 
optimal for selectively detecting the Raman signal from the epithelial tissue. The above result indicates that Raman probe designs with a proper selection of the refractive index and diameter of the ball lens can improve both the collection efficiency and the ability to preferentially acquire the Raman photons emitted from the epithelium layer of epithelial tissue.

\subsection{Effect of the probe-tissue distance on tissue Raman measurements}

In practical tissue measurements, the probe-to-tissue distance may play a significant role in depth-resolved tissue spectroscopy [10, 11, 14, 15]. We comprehensively study the relationship of the collection efficiency of the ball lens Raman probe designs versus probetissue distances. Fig 12a shows an example of the collection efficiency versus probe-tissue distances using the Raman probe with the ball lens' diameter of $3 \mathrm{~mm}$ and refractive index of 1.83 . The collection efficiency drops sharply with the increased probe-tissue distances $(0$ to $200 \mu \mathrm{m}$ ) and then the collection efficiency gradually decreases to a minimum (250 to 1000 $\mu \mathrm{m})$. We also study the effect of the probe-tissue distance on the ratio of the Raman signal collected from the epithelium layer to the overall Raman signal from the entire epithelial tissue in depth-resolved Raman measurements (Fig. 12b). It is found that when the probe-totissue distances are in the range of 0 to $100 \mu \mathrm{m}$, Raman photons arising from the tissue epithelium layer account for over $90 \%$ of the total Raman photons of the entire epithelial tissue. The above results suggest that the collection efficiency and the depth-selectivity of the Raman probe design can be optimized by setting a proper gap between the probe and the tissue.
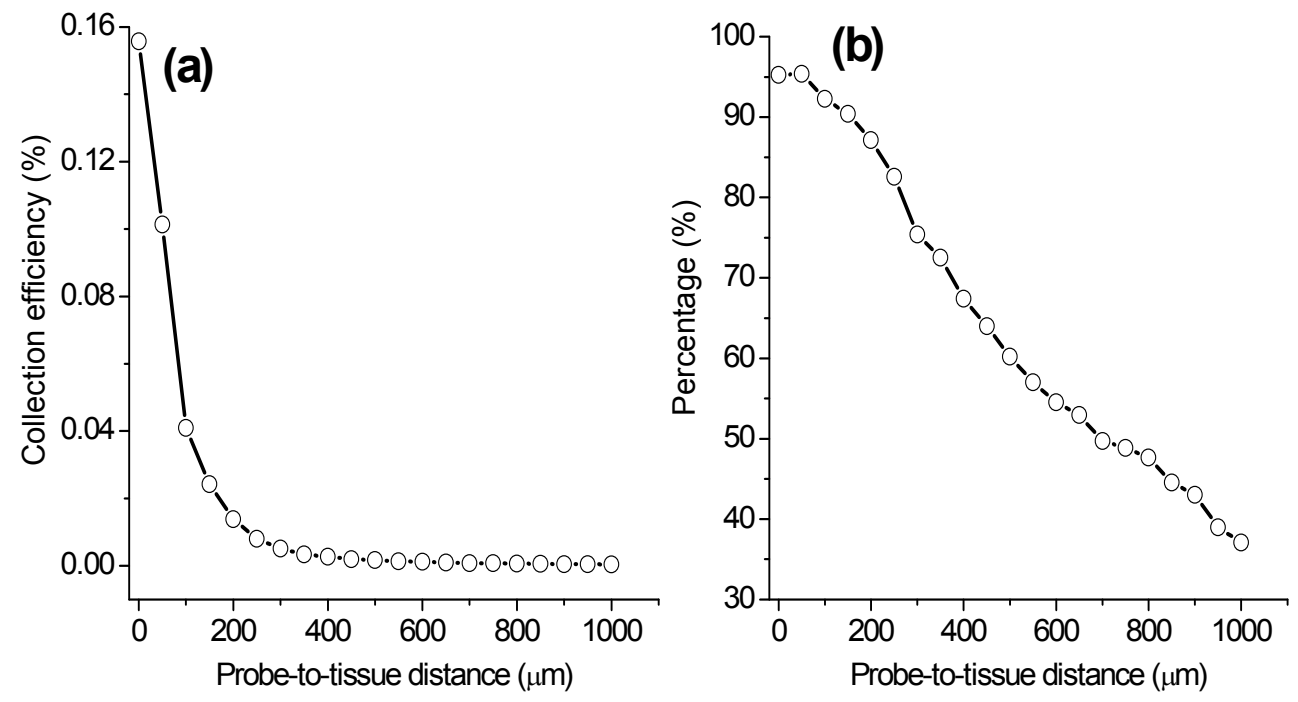

Fig. 12. (a) Collection efficiency of the Raman probe as a function of probe-tissue distances; (b) Percentage of the Raman signal collected from the epithelium layer to the overall Raman signal from the entire epithelial tissue. The refractive index and the diameter of the ball lens are 1.83, and $3 \mathrm{~mm}$, respectively, in Raman probe design. 


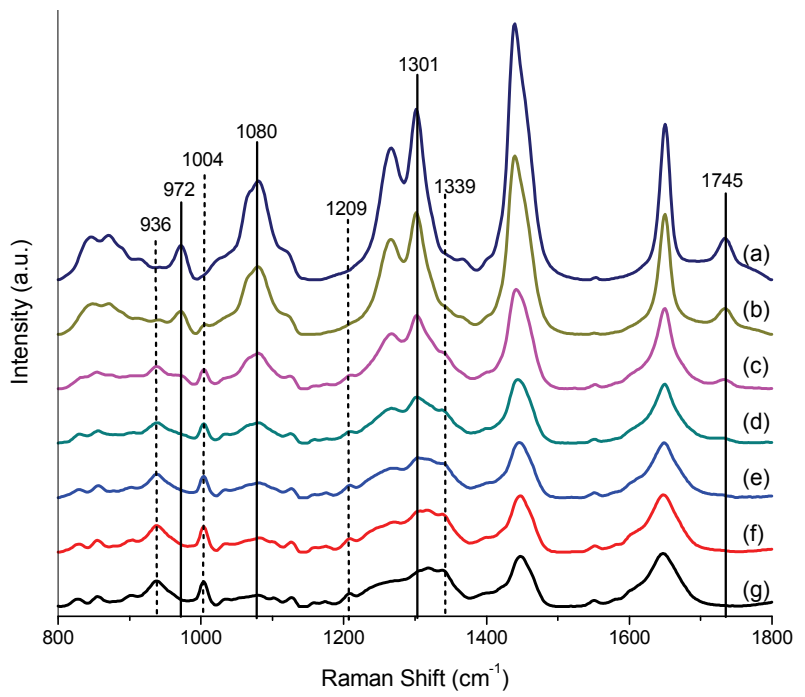

Fig. 13. Raman spectra acquired from chicken muscle and fat tissue, as well as from the twolayer tissue phantoms with the muscle tissue thickness of $0.3,1.2,2.1,3$ and $3.9 \mathrm{~mm}$, respectively. Spectra: (a) fat tissue; (b)-(f): two-layer tissue phantoms with the muscle tissue layer of thickness of $0.3,1.2,2.1,3.0$ and $3.9 \mathrm{~mm}$, respectively, overlaying on a fat tissue layer (thickness of $5 \mathrm{~mm}$ ); (g) muscle tissue. Note that all tissue Raman spectra are acquired with an integration time of $1 \mathrm{~s}$ under the $785-\mathrm{nm}$ excitation power of $1.5 \mathrm{~W} / \mathrm{cm}^{2}$. The dotted and solid lines indicated in Raman spectra stand for the distinctive Raman peaks originating from the muscle and fat chicken tissue, respectively.

\subsection{Raman experiments on two-layer tissue phantom}

We have also carried out tissue Raman measurements on a two-layer tissue phantom to evaluate the performance of a ball lens Raman probe design using a sapphire ball lens (refractive index of 1.76, and a diameter of $3 \mathrm{~mm}$ ). The two-layer tissue phantom was constructed by overlaying different thicknesses of chicken muscle tissue on a chicken fat tissue: the thickness of fat tissue layer was fixed to be $5 \mathrm{~mm}$, while the thickness of the muscle tissue layer varied from 0.3 to $3.9 \mathrm{~mm}$. Fig. 13 shows Raman spectra acquired from chicken muscle and fat tissue, as well as from the two-layer tissue phantoms with the muscle tissue thickness of $0.3,1.2,2.1,3$ and $3.9 \mathrm{~mm}$, respectively. It is observed that chicken muscle and fat tissue show distinctively different Raman spectral features. For instance, the Raman spectrum of muscle tissue shows distinct Raman peaks at 936 (C-C stretching of proteins), 1004 (C-C stretching of phenylalanine), 1209 (C- $\mathrm{C}_{6} \mathrm{H}_{5}$ stretching of tryptophan and phenylalanine), and $1339 \mathrm{~cm}^{-1}\left(\mathrm{CH}_{3} \mathrm{CH}_{2}\right.$ wagging of collagen). In contrast, the Raman spectrum of fat tissue exhibits four different unique peaks at 972 (Calcium-phosphate stretching of cholesterols/lipids), 1080 (C-C/C-O stretching of phospholipids), $1301\left(\mathrm{CH}_{2}\right.$ twisting and wagging modes of lipids, triglycerides (fatty acids)) and $1745 \mathrm{~cm}^{-1}(\mathrm{C}=\mathrm{O}$ stretching of ester (phospholipids)) [2, 8]. With the thickness of muscle tissue layer increasing from 0 to $3.9 \mathrm{~mm}$ in the two-layer tissue phantom, the Raman intensity at 1004 $\mathrm{cm}^{-1}$ for muscle tissue increases by 2.1 to 5.6 folds, whereas the Raman signal at $1745 \mathrm{~cm}^{-1}$ for fat tissue undergoes a 28 to 1.6 fold reduction in intensity (Fig. 14). This implies that the 
Raman signal contribution of the surface muscle layer to the overall Raman signal increases as the muscle tissue thickness increases, while the signal contribution of the deeper fat tissue layer reduces concomitantly. The results above indicate the potential of the ball lens Raman probe design for effective depth-selective Raman measurements in layered tissue.

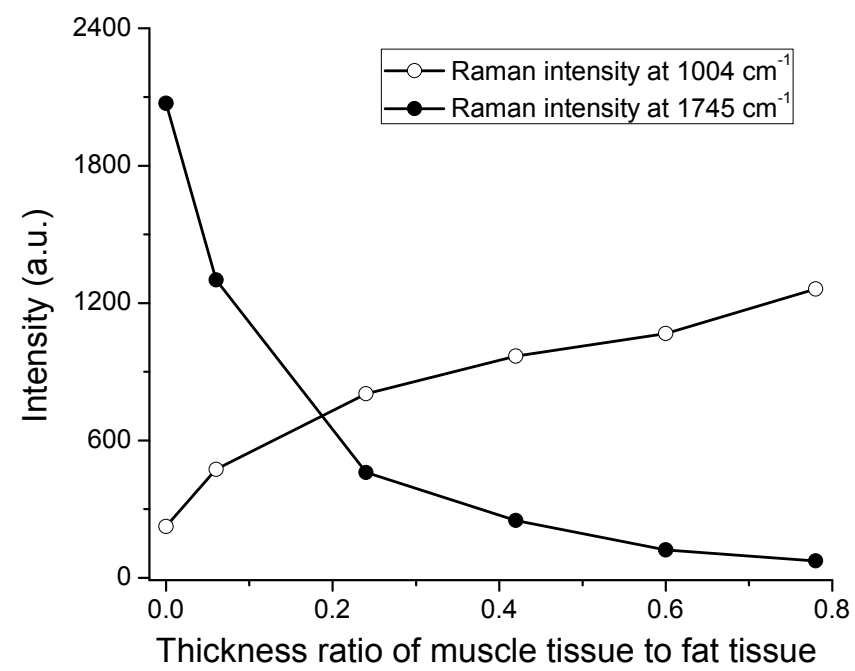

Fig. 14. Raman peaks intensities at 1004 and $1745 \mathrm{~cm}^{-1}$ as a function of thickness ratios of the muscle tissue layer to the fat tissue in a two-layer tissue phantom.

\section{Conclusions}

We have proposed a fiber-optic Raman probe coupled with a ball lens for improving depthresolved Raman measurements of epithelial tissue. We have comprehensively evaluated the effects of optical configurations of the ball lens Raman probe design on depth-resolved Raman measurements using Monte Carlo simulations. The MC calculation results show that depth discriminations of Raman signals are achievable by changing either the refractive index or diameter of the ball lens, or the ball lens-tissue distances. The Raman probe design by increasing the refractive index or reducing the diameter of the ball lens is in favor of probing Raman signals particularly arising from the shallower regions of the epithelium layer of tissue. Experimental evaluation of a ball lens probe design confirms its ability for efficient depth-resolved measurement on layered tissues. The simulations results can serve as a guideline to optimize the optical configurations of the Raman probe design aiming at improving the detection efficiency of layered tissue Raman measurements, which may facilitate the applications of depth-resolved Raman technique in early diagnosis and detection of precancer or early cancer at the molecular level.

\section{Acknowledgements}

This work was supported by the Biomedical Research Council, the National Medical Research Council, and the Faculty Research Fund from the National University of Singapore. 


\section{References}

[1] N. Stone, C. Kendall, N. Shepherd, P. Crow, and H. Barr, "Near-infrared Raman spectroscopy for the classification of epithelial pre-cancers and cancers," J. Raman Spectrosc. 33, 564-573 (2002).

[2] Z. Huang, A. McWilliams, H. Lui, D. I. McLean, S. Lam, and H. Zeng, "Near-infrared Raman spectroscopy for optical diagnosis of lung cancer," Int. J. Cancer 107, 10471052 (2003).

[3] Z. Huang, H. Zeng, I. Hamzavi, D. I. McLean, and H. Lui, "Rapid near-infrared Raman spectroscopy system for real-time in vivo skin measurements," Opt. Lett. 26, 17821784 (2001).

[4] M. G. Shim, B. C. Wilson, E. Marple, and M. Wach, "Study of fiber-optic probes for in vivo medical Raman spectroscopy," Appl. Spectrosc. 53, 619-627 (1999).

[5] Z. Huang, S. K. Teh, W. Zheng, J. Mo, K. Lin, X. Shao, K. Y. Ho, M. Teh, and K. G. Yeoh, "Integrated Raman spectroscopy and trimodal wide-field imaging techniques for real-time in vivo tissue Raman measurements at endoscopy," Opt. Lett. 34, 758-760 (2009).

[6] R. Drezek, C. Brookner, I. Pavlova, I. Boiko, A. Malpica, R. Lotan, M. Follen, and R. Richards-Kortum, "Autofluorescence microscopy of fresh cervical-tissue sections reveals alterations in tissue biochemistry with dysplasia\&para," Photochem. Photobiol. 73, 636-641 (2001).

[7] F. Abdul-Karim, Y. S. Fu, J. W. Reagan, and W. B. Wentz, "Morphometric study of intraepithelial neoplasia of the uterine cervix," Obstet. Gynecol. 60, 210-214 (1982).

[8] J. Mo, W. Zheng, J. J. H. Low, J. Ng, A. Ilancheran, and Z. Huang, "High wavenumber Raman spectroscopy for in vivo detection of cervical dysplasia," Anal. Chem. 81, 8908-8915 (2009).

[9] W. C. Shih, K. L. Bechtel, and M. S. Feld, "Intrinsic Raman spectroscopy for quantitative biological spectroscopy part I: theory and simulations," Opt. Express 16, 1272612736 (2008).

[10] T. J. Pfefer, K. T. Schomacker, M. N. Ediger, and N. S. Nishioka, "Light propagation in tissue during fluorescence spectroscopy with single-fiber probes," Selected Topics in Quantum Electronics, IEEE Journal of 7, 1004-1012 (2001).

[11] T. J. Pfefer, K. T. Schomacker, M. N. Ediger, and N. S. Nishioka, "Multiple-fiber probe design for fluorescence spectroscopy in tissue," Appl. Opt. 41, 4712-4721 (2002).

[12] L. Nieman, A. Myakov, J. Aaron, and K. Sokolov, "Optical sectioning using a fiber probe with an angled illumination-collection geometry: evaluation in engineered tissue phantoms," Appl. Opt. 43, 1308-1319 (2004).

[13] R. A. Schwarz, D. Arifler, S. K. Chang, I. Pavlova, I. A. Hussain, V. Mack, B. Knight, R. Richards-Kortum, and A. M. Gillenwater, "Ball lens coupled fiber-optic probe for depth-resolved spectroscopy of epithelial tissue," Opt. Lett. 30, 1159-1161 (2005).

[14] F. Jaillon, W. Zheng, and Z. Huang, "Beveled fiber-optic probe couples a ball lens for improving depth-resolved fluorescence measurements of layered tissue: Monte Carlo simulations," Phys. Med. Biol. 53, 937-951 (2008).

[15] F. Jaillon, W. Zheng, and Z. Huang, "Half-ball lens couples a beveled fiber probe for depth-resolved spectroscopy: Monte Carlo simulations," Appl. Opt. 47, 3152-3157 (2008). 
[16] J. T. Motz, M. Hunter, L. H. Galindo, J. A. Gardecki, J. R. Kramer, R. R. Dasari, and M. S. Feld, "Optical Fiber Probe for Biomedical Raman Spectroscopy," Appl. Opt. 43, 542554 (2004).

[17L. Wang, S. L. Jacques, and L. Zheng, "MCML--Monte Carlo modeling of light transport in multi-layered tissues," Comput. Methods Programs Biomed. 47, 131-146 (1995).

[18] A. J. Welch, C. Gardner, R. Richards-Kortum, E. Chan, G. Criswell, J. Pfefer, and S. Warren, "Propagation of fluorescent light," Lasers Surg. Med. 21, 166-178 (1997).

[19] G. M. Palmer, and N. Ramanujam, "Monte-Carlo-based model for the extraction of intrinsic fluorescence from turbid media," J. Biomed. Opt. 13, 024017-024019 (2008).

[20] W. C. Shih, K. L. Bechtel, and M. S. Feld, “Intrinsic Raman spectroscopy for quantitative biological spectroscopy part I: theory and simulations," Opt. Express 16(17), 1272612736 (2008).

[21] K. L. Bechtel, W. C. Shih, and M. S. Feld, "Intrinsic Raman spectroscopy for quantitative biological spectroscopy part II: experimental applications," Opt. Express 16(17), 12737-12745 (2008).

[22] L. G. Henyey, and J. L. Greenstein, "Diffuse radiation in galaxy," Astrophys. J 93, 70-83 (1941).

[23] M. J. Riedl, Optical design fundamentals for infrared systems (SPIE Press, Bellingham, Wash., 2001).

[24] S. K. Chang, D. Arifler, R. Drezek, M. Follen, and R. Richards-Kortum, "Analytical model to describe fluorescence spectra of normal and preneoplastic epithelial tissue: comparison with Monte Carlo simulations and clinical measurements," J. Biomed. Opt. 9, 511-522 (2004). 


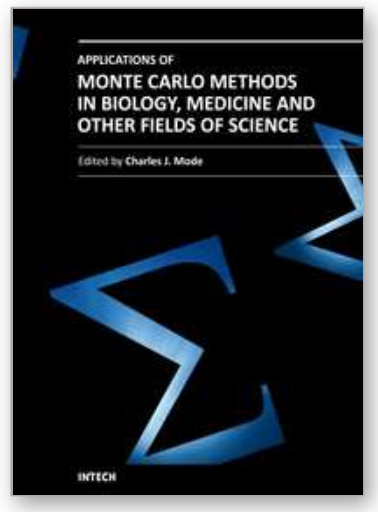

\section{Applications of Monte Carlo Methods in Biology, Medicine and Other Fields of Science}

Edited by Prof. Charles J. Mode

ISBN 978-953-307-427-6

Hard cover, 424 pages

Publisher InTech

Published online 28, February, 2011

Published in print edition February, 2011

This volume is an eclectic mix of applications of Monte Carlo methods in many fields of research should not be surprising, because of the ubiquitous use of these methods in many fields of human endeavor. In an attempt to focus attention on a manageable set of applications, the main thrust of this book is to emphasize applications of Monte Carlo simulation methods in biology and medicine.

\section{How to reference}

In order to correctly reference this scholarly work, feel free to copy and paste the following:

Zhiwei Huang (2011). Fiber-optic Raman Probe Coupled with a Ball Lens for Improving Depth-resolved Raman Measurements of Epithelial Tissue: Monte Carlo Simulations, Applications of Monte Carlo Methods in Biology, Medicine and Other Fields of Science, Prof. Charles J. Mode (Ed.), ISBN: 978-953-307-427-6, InTech, Available from: http://www.intechopen.com/books/applications-of-monte-carlo-methods-in-biology-medicineand-other-fields-of-science/fiber-optic-raman-probe-coupled-with-a-ball-lens-for-improving-depth-resolvedraman-measurements-of-

\section{INTECH}

open science | open minds

\section{InTech Europe}

University Campus STeP Ri

Slavka Krautzeka 83/A

51000 Rijeka, Croatia

Phone: +385 (51) 770447

Fax: +385 (51) 686166

www.intechopen.com

\section{InTech China}

Unit 405, Office Block, Hotel Equatorial Shanghai

No.65, Yan An Road (West), Shanghai, 200040, China

中国上海市延安西路65号上海国际贵都大饭店办公楼 405 单元

Phone: +86-21-62489820

Fax: $+86-21-62489821$ 
(C) 2011 The Author(s). Licensee IntechOpen. This chapter is distributed under the terms of the Creative Commons Attribution-NonCommercialShareAlike-3.0 License, which permits use, distribution and reproduction for non-commercial purposes, provided the original is properly cited and derivative works building on this content are distributed under the same license. 